\title{
A Segmentation Method to Improve Iris-based Person Identification
}

\author{
Asheer K.Bachoo \\ Department of Computer Science \\ University of KwaZulu-Natal \\ Durban, 4041, \\ South Africa \\ Email: Bachooa@ukzn.ac.za
}

\author{
Jules-R Tapamo \\ Department of Computer Science \\ University of KwaZulu-Natal \\ Durban, 4041, \\ South Africa \\ Email: tapamoj@ukzn.ac.za
}

\begin{abstract}
The authentication of individuals using irisbased recognition is a widely developing technology. Precise and unobtrusive image acquisition is not always possible. This introduces a number of ill-affecting factors to the subsequent localisation and characterisation stages. Common problems include variations in lighting, poor image quality, noise and interference caused by eyelashes. The feature extraction and classification procedures rely heavily on the rich textural details of the iris to provide a unique digital signature for an individual. As a result, the stability and integrity of a system depends on effective segmentation of the iris to generate the iris-code. The previously mentioned problems hinder this task. A new segmentation method is presented to tackle these problems. Principal component analysis (PCA) is discussed as a preprocessing technique that removes redundant and useless data. Variations in lighting and noise are handled using an application of median filtering and adaptive thresholding. Finally, edge detection and the Hough transform are discussed for locating the circular boundaries of the pupil and sclera.
\end{abstract}

keywords:Person Identification, Principal component analysis, Haar Transform, segmentation

\section{INTRODUCTION}

In recent times, our security has been threatened by terrorist groups and malicious individuals, highlighting the need for robust and secure person identification. The automated authentication of individuals is a complex and sensitive task. Current methods of authentication - such as passwords and electronic cards - are susceptible to fraud and theft.

An approach to this problem is an automated biometric identification system [1]- a system that uses a unique attribute of the human body to es- tablish a digital signature for recognition of the individual. Our increasing knowledge of the human body's physiology has developed a number of authentication technologies - retinal, fingerprint, hand geometry, facial, voice, odour and iris systems. The most common ones are discussed.

The primary biometric is fingerprint identification. Ridge bifurcations and ridge endings are localised during the processing stage. These characteristics are used to generate an orientation field of the fingerprint, which subsequently provides the discriminating details for authentication of persons. Fingerprint systems are favourable for their low cost and minimum space requirement.

Authentication by hand geometry has gained widespread interest. The feature vector incorporates information concerning the length of the fingers and hand shapes. The drawback of these implementations concerns their relatively high false accept rates (FAR) and false reject rates (FRR).

Authentication by facial appearance is perhaps the most logical yet complex of the biometrics. Facial images can be captured quite easily and stored in large databases for retrieval. However, the authentication stage is hindered by aspects such as aging and facial expression (physical), lighting and camera rotation. A general solution to these problems has yet to be presented.

Voice (speaker) recognition exploits the differences in physiological and behavioural aspects of the human speech reduction system. The speech waves contain spectral components that are analysed for unique features. This system is also susceptible 
to a number of problems such as imposters, noise and physiological changes of the person.

Iris recognition is a relatively new yet powerful solution to all the problems presented above. It has low FRRs and FARs and the digital signature established is highly unique. Replication of the iris and fraudulent efforts are highly unlikely.

\section{BACKGROUND}

The iris [2] begins its formation in the 3rd month of gestation. By the 8th month, its distinctive pattern is complete. However, pigmentation and even pupil size increase as far up as adolescence [3].

The iris is a multilayered texture. This combination of layers and colour provide a highly distinctive pattern. An assortment of texture variations is possible. They include:

- Contractile lines related to the state of the pupil

- Crypts - irregular atrophy of the border layer

- Naevi - small elevations of the border layer

- Freckles - collections of chromataphores

- Colour variation - an increase in pigmentation yields darker coloured irides.

Of the utmost importance in a biometric identification system is the stability and uniqueness of the object being analysed. Ophthalmologists [4] and anatomists [5], during the course of clinical observations, have noted that the irises of individuals are highly distinctive. This extends to the left and right eye of the same person. Repeated observations over a period of time have highlighted little variation in the patterns.

Developmental biology has also provided evidence [3]. Although the general structure of the iris is genetically determined, the uniqueness of its minutae is highly dependent on circumstances. As a result, replication is almost impossible. It has also been noted that, following adolescence, the iris remains stable and varies little for the remainder of the person's life. Development is continuous during the early and adolescent years (pigmentation continues as well as an increase in pupil size) [5], [3].

In 1936, Frank Burch, an ophthalmologist, proposed the idea of using iris patterns for personal identification. However, this was only documented by James Doggarts in 1949. The idea of iris identification for automated recognition was finally patented by Aran Safir and Leonard Flom in 1987. Although they had patented the idea, the two ophthalmologists were unsure as to a practical implementation for the system. They commissioned John Daugman to develop the fundamental algorithms in 1989. These algorithms were patented by Daugman in 1994 and now form the basis for all current commercial iris recognition systems. The Daugman algorithms are owned by Iridian Technologies and they are licensed to several other companies [3].

\section{PREVIOUS WORKS}

The fundamental iris algorithm and system was developed by John Daugman. This was presented in his landmark paper [6] and subsequently updated[7]. The iris is segmented using integro-differential operators. To extract the rich details of the texture, he uses complex-valued 2D Gabor wavelets to extract discriminating information. Recognition is done by means of a test of statistical independence for two iris codes. A failure of the test implies a match. The matching system implements a normalized Hamming distance criteria

Wildes et al. [8] also describe an efficient and consistent algorithm. The system uses edge maps and a Hough transform to locate the region of interest. Feature extraction is performed with an application of Laplacian of Gaussian filters at different resolutions. A normalized correlation between the acquired iris representation and the stored one is employed for pattern matching.

Boles [9] system extracts features using zerocrossing representation of a $1 \mathrm{D}$ wavelet transform. Ali and Hassanien [10] perform feature extraction by means of the Haar wavelet. Their system achieved a rate comparable to Daugman's and Wildes. Lim et al. [11] designed a system that combines the features of the Haar wavelet and a neural network to achieve recognition. Ma et al. [12] consider multi-channel Gabor filtering to extract texture details.

There have also been a number of other interesting ideas for iris recognition. MICA (Multiresolution Independent Component Analysis) [13] and an application of the Hough transform for iris identification [14] are well documented. A promising avenue has been an analysis of the Fourier spectra of the optical transmission binary models of human 
irises [?]. The interested reader can also survey literature that addresses circular symmetric filters for recognition purposes [15]

\section{IRIS SEGMENTATION}

Segmentation of the iris from an image of the eye is sensitive to numerous factors - noise, uneven lighting and eyelid and eyelash interference. Although the pupil and iris borders can be modelled using two non-concentric circles, with the larger one forming a closed contour around the smaller one, detection of these two boundaries is not a simple problem. In a typical case, the outer iris boundary is a very soft gradient that standard edge functions do not detect. This boundary is sometimes only partially visible or, possibly covered by eyelash.

The first stage in the proposed algorithm uses principal component analysis (PCA) - a statistical approach that transforms the image vector space. The algorithm is adapted from [16]. We need to first establish the covariance matrix of the image and then determine its eigenvectors. These eigenvectors are associated with the directions of the principle components of the original data. Their statistical importance is denoted by the corresponding eigenvectors. The specific algorithm is as follows:

1) Given an and multi-band $N \times M$ image I defined as follows:

$$
\mathrm{I}=\left[\begin{array}{cccc}
x_{11} & x_{12} & \ldots & x_{1 M} \\
x_{21} & x_{22} & \ldots & x_{2 M} \\
\vdots & \vdots & \ddots & \vdots \\
x_{N 1} & x_{N 2} & \ldots & x_{N M}
\end{array}\right]
$$

where $1 \leq i \leq N$ and $1 \leq j \leq M$ in other words $x_{i j}$ is a pixel consisting of three bands : RED, GREEN and BLUE.

2) Compute the mean $\bar{x}=\left(\overline{x^{R}}, \overline{x^{G}}, \overline{x^{B}}\right)$ (where $\overline{x^{k}}=\frac{1}{N \times M} \sum_{i=1}^{N} \sum_{j=1}^{M} x_{i j}^{k}$ for $k \in$ $\{R, G, B\})$

3) for $i, j \in\{R, G, B\}$ calculate

$$
C_{i j}=\frac{1}{N \times M} \sum_{k=1}^{N} \sum_{l=1}^{M}\left(x_{k l}^{i}-\overline{x^{i}}\right)\left(x_{k l}^{j}-\overline{x^{j}}\right)
$$

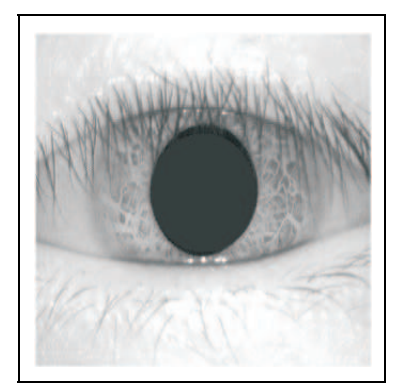

(a)

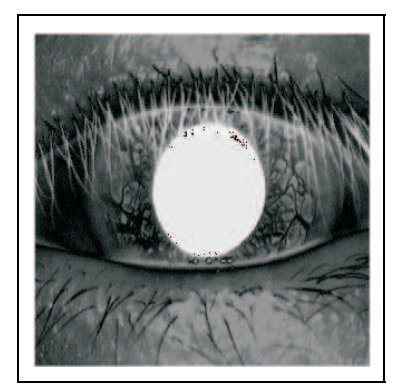

(b)
Fig. 1. (a):Original image (b): Principal component.

and derive the covariance matrix $C=$ $\left(C_{i j}\right)_{i, j \in\{R, G, B\}}$

4) Calculate the eigenvalues $\lambda_{i}$ and eigenvectors $e_{i}$ of $\mathrm{C}$ such that

$$
\left(C-\lambda_{i} I\right) e_{i}=0 \text { for } i=1,2,3
$$

$5)$ Sort the eigenvectors in descending order according to their corresponding eigenvalues

6) Create the transformation matrix $T$ with the sorted vectors as the rows

$$
T=\left[\begin{array}{l}
e_{1} \\
e_{2} \\
e_{3}
\end{array}\right]=\left[\begin{array}{lll}
e_{11} & e_{12} & e_{13} \\
e_{21} & e_{22} & e_{23} \\
e_{31} & e_{32} & e_{33}
\end{array}\right]
$$

7) Compute the Transform $\mathrm{P}$ of $\mathrm{I}$ as follows: $P=T \bullet I=\left(P_{1}, P_{2}, P_{3}\right)$

8) From each pixel, we select the first band, which is the first component, represented by $P_{1}$.

The transformed pixels in the new vector space are void of any physical meaning. They do not correspond to a colour representation that can be visualised. The result of the transformation is an enhanced image - soft sclera edges are more pronounced. If a centre of mass model is computed based on the image histogram, the centre corresponds to the density information supplied by the pupil and eyelash regions. This is a common characteristic of iris images. As a result, the PCA algorithm aligns and transforms the vector space so as to enhance the edge information of the iris.

Noise removal is performed on the PCA image using the median filter [17]. The median filter is a non-linear smoothing technique. The current pixel is replaced by the median of the brightness of its neighbourhood. This reduces any speckle present in the image. 


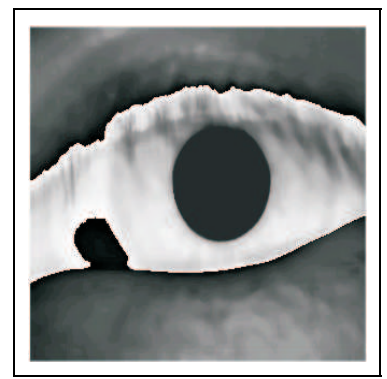

(a)

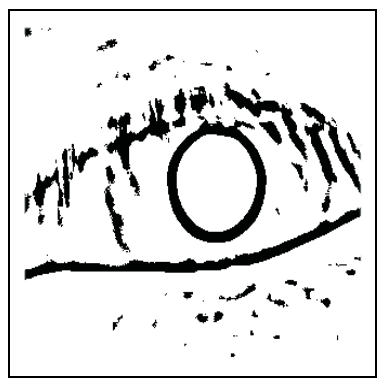

(b)
Fig. 2. (a):median filtered of fig 1.b (b): Adaptive threshold of fig 2.a.

To facilitate the segmentation process, the image is thereafter thresholded to separate the foreground from the background. Global thresholding segments the pupil . To maximise the effectiveness of the PCA algorithm, an adaptive thresholding algorithm [17] is executed for iris separation. Global thresholding computes the threshold constant using the image space. Global thresholding transforms an image $f$ to a binary image $g$ as follows:

$$
g(x, y)= \begin{cases}1 & \text { if } f(x, y) \geq T \\ 0 & \text { otherwise }\end{cases}
$$

$T$ is the threshold (a brightness constant). For effectiveness, the histogram must display two dominant peaks, with the threshold calculated as a value lying in the valley between them. Preliminary analysis of the image data reveals an optimal peak corresponding to the pixels of the pupil, which makes global thresholding suitable for pupil localisation.

In most real world scenarios, variations in lighting and noise cause fluctuations in the image histogram. A dynamic approach is required to compute a threshold in a localised zone of the image. This is typically a window of size $b b$. An effect method uses the mean neighbourhood function to perform adaptive thresholding. The threshold at pixel $(i, j)$ is calculated as

$$
T(i, j)=m(i, j)+C
$$

where $C \in \mathbb{R}$ and $m(i, j)$ is the local sample mean determined within the $b \times b$ window. The windowing function effectively segments the iris, detecting important edge information.

After thresholding, edges are located using Canny edge detection [18]. This algorithm resolves borders

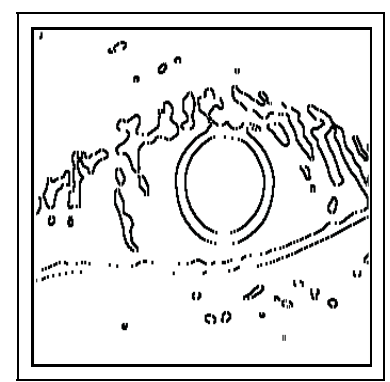

(a)

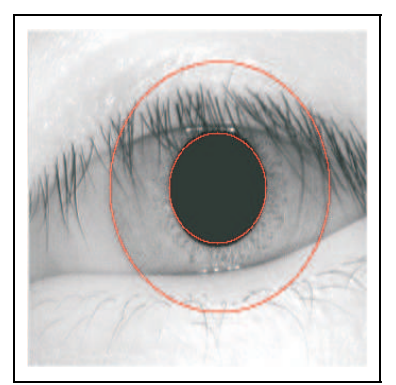

(b)
Fig. 3. (a):edge map of fig 1.a (b): Hough transform.

into lines that are a single pixel thick, which greatly reduces the search space for the Hough transform, improving overall performance. The iris is precisely localised using the Hough transform.

The Hough transform [17], [19] locates contours in an $n$-dimensional parameter space by examining whether they lie on curves of specified shape. It represents an image $I$ in terms of an accumulator array A, defined as follows

$$
A: P \rightarrow N \text { where } P=P_{1} \times P_{2} \times \ldots \times P_{\rho}
$$

where $\rho$ determines the range of $i$-parameters of a $\rho$ - dimensional space $P$. $A$ is determined through the computation of partial values for points of $I$ and adding them to previous ones. Peaks in the accumulator array correspond to curves of a desired shape.

$$
(x-a)^{2}+(y-b)^{2}=r^{2}
$$

defines a circle of radius $r$ and center $(a, b)$ in $x y$-space. The accumulator array is 3-dimensional $(a, b, r)$ - and for each edge pixel $(x, y)$ we update the accumulator cell with parameters $(a, b, r)$ that passes through $(x, y)$. The binary images from the thresholding stage provide the edge data.

Using the Hough transform circles are mapped to the binary images for tracking of the outer pupil and iris boundaries. Firstly, the centre co-ordinates and radius of the pupil is established. Thereafter, these parameters are used as references for the computation of the iris boundary parameters.

\section{TRANSFORMATION FROM CARTESIAN TO POLAR COORDINATES}

Once the region of interest has been isolated, it is transformed to a dimensionless polar system. 


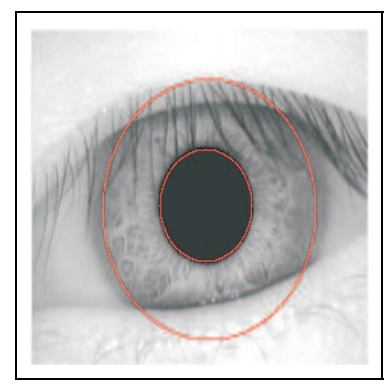

(a)

Fig. 4. (a):region of interest (b): transformed region.

This form is standard irrespective of iris size, pupil diameter or resolution. The implemented algorithm is based on Daugman's [6] stretched polar coordinate system. The idea behind the dimensionless polar system is to assign an $r$ and $\theta$ value to each co-ordinate in the iris that will remain invariant to the possible stretching and skewing of the image.

For our transformation, the $r$ value ranges from $[0,1]$ and the angular value spans the normal $[0,2 \pi]$. The remapping is done according to the following formulas:

$$
\begin{gathered}
\left\{\begin{array}{l}
x(\rho, \theta)=(1-\rho) x_{\rho}(\theta)+\rho x_{i}(\theta) \\
y(\rho, \theta)=(1-\rho) y_{\rho}(\theta)+\rho y_{i}(\theta)
\end{array}\right. \\
\left\{\begin{array}{l}
x_{\rho}(\theta)=x_{\rho 0}(\theta)+r_{\rho} \sin (\theta) \\
y_{\rho}(\theta)=y_{\rho 0}(\theta)+r_{\rho} \sin (\theta)
\end{array}\right. \\
\left\{\begin{array}{l}
x_{i}(\theta)=x_{i 0}(\theta)+r_{i} \sin (\theta) \\
y_{i}(\theta)=y_{i 0}(\theta)+r_{\rho} \sin (\theta)
\end{array}\right.
\end{gathered}
$$

$\left(x_{\rho 0}, y_{\rho 0}\right)$ is the centre of the pupil and $\left(x_{i 0}, y_{i 0}\right)$ is the centre of the iris. $r_{\rho}$ is the radius of the pupil and $r_{i}$ is the radius of the iris. $x_{\rho}$ and $x_{i}$ are the coordinates of points bordering the pupil's and the iris' radii. The remapping is done so that the transformed image is a rectangle with dimension $512 \times 64$. Figure $4 . b$ shows the result of this transform

\section{IRIS AUTHENTICATION}

To extract the rich details of the iris texture, the Haar wavelet [20] decomposes and analyses the signal at different scales and frequencies.

The texture is mapped to a grid of $512 \times 64$ pixels. A generalised Haar[21], [22] algorithm decomposes this texture band, extracting 128 feature bits for the iris code. Our transformed image has dimensions that are a power of two $-2^{n} \times 2^{m}$. The reason for this is that the Haar transform we are implementing requires us to divide the image into non-overlapping blocks of $2 \times 2$ pixels. We perform high-pass and low-pass filtering on these blocks and create a new image from the coefficients. Thereafter, we recurse through a sub-block of the new image.

Codes are matched using a normalized Hamming distance [20]. It is determined using the exclusiveor (XOR) boolean function. If $\mathrm{A}$ and $\mathrm{B}$ are two codes that we are matching - with $A_{j}$ and $B_{j}$ being corresponding bits - the Hamming distance function is defined as follows;

$$
H D=\frac{1}{128} \sum_{j}^{128} A_{j} \oplus B_{j}
$$

A hamming distance of 0 implies a perfect match while 1 implies codes that are not similar at all. The response between two bits is equally likely to be a 0 or 1 , so the average hamming distance between two codes is 0.5 .

In order to recognize an individual, it is essential that a threshold be established for the Hamming distance criterion. A Hamming distance below this threshold implies that the 2 irises are the same and a match has been found. The selected threshold is 0.375 .

The possibility of two irises having the same vectors is very low. The match of only $62.25 \%$ of its bits provides a high confidence match for the iris recognition system. While the match rate may be low, it is also important that the FAR and FRR are low. The system achieved this with high confidence.

\section{EXPERIMENT RESULTS}

The system was tested on images supplied by Institute of Automation, Chinese Academy of Sciences, from their CASIA iris database. Fifteen sets of images were selected for testing. Each set consisted of 7 images of a particular individual. An image was selected from each set to create an iris-code template and the remaining images were matched against these templates. The results were as follows: 


\begin{tabular}{|l|r|}
\hline Matches & $52.2 \%$ \\
\hline False rejects & $43.3 \%$ \\
\hline False matches & $4.5 \%$ \\
\hline
\end{tabular}

The following three issues have been identified as problems in the system that needs to be addressed for better results:

1) Eyelash interference - Iris images obscured by eyelash are difficult to segment. Their texture details are obscured and texture analysis and code generation under these conditions proves unsuccessful during the recognition stage. Recent work conducted in [23] addresses this problem and a future investigation will be undertaken.

2) Rotation of the region of interest - Rotation of the iris has proved to be another stumbling block. Numerous images demonstrate rotation and even tilt and this plays a role in the low recognition rate achieved. A solution to this is to use Euler matrices for rotational invariance. Daugman [7] suggests a bit-wise shift of the iris code during the matching process and a subsequent analysis to decide on a best match.

3) Poor image quality - A number of the iris images have poor texture quality and detail. We can only hope to improve the image acquisition stage to solve this problem.

\section{FUTURE WORKS}

The results of the experiment demonstrate the effectiveness of the proposed algorithm in detecting soft gradients at the iris borders. However, an improved Hough transform is required since this is the most computationally intensive part of the implementation. Another deciding factor in the robustness of the system is the presence of eyelashes, which introduces misleading data into the edge image and the extracted texture region. Segmentation of eyelashes will be studied in a future investigation.

\section{Conclusion}

A new method has been presented for iris segmentation. Principle component analysis (PCA) and adaptive thresholding are very effective as a preprocessing step prior to the contour detection stage. Experimental results with authentication using haar wavelets demonstrated that our approach is promising to improve iris-based person identication.

\section{REFERENCES}

[1] R. M. Senior Ratha N.K and Bolle R.M., "Automated biometrics," in ICAPR, 2001.

[2] Adler F.H., Phylosophy of the eye, MO:Mosby, St. Louis, 1965.

[3] P.C. kronfeld, "The gross and embryology of the eye"," The Eye, vol. 1, pp. 1-66, 1968.

[4] Flom L. and A. Safir, "Iris recognition system," 1987.

[5] Davson H., Davson's Phylosophy of the eye, MacMillan, London, 1990.

[6] J. Daugman, "High confidence visual recognition of persons by a set of statistical independance," IEEE Trans. Pattern Analysis Mach. Intell., vol. 15, no. 11, pp. 1148-1961, 1993.

[7] J. Daugman, "The importance of being random: stastical principles of iris recognition," Pattern Recognition, vol. 36, no. 2, pp. 279-291, 2003.

[8] Wildes L.P., "Iris recognition: An emerging biometric technology," Proceedings of the IEEE, vol. 85, no. 9.

[9] Boles L.P. and Boashash B., "A human identification technique using images of the iris and wavelet transform," IEEE Trans. on Signal Processing, vol. 46, no. 4.

[10] Ali J.M.H and A.E Hassanien, "An iris recognition system to enhance e-security environment based on wavelet theory," Advanced Modelling and Optimization, vol. 5, no. 2.

[11] O. Byeon Lim K., K. Lee and T. Kim, "Efficient iris recognition through improvement of feature vector and classifier," ETRI Journal, vol. 23, no. 2, pp. 61-70, 2001.

[12] Y. Wang Lim K. and T. Tan, "Iris recognition based on multichanel gabor filtering," in Fifth Asian Conference on Computer Vision, 2002, vol. 1, pp. 279-283.

[13] S Noh Bae K. and J. Kim, "Iris feature extraction using independent component analysis," in AVBPA, 2003, pp. 838844.

[14] Blackledge J. Zorski W., Foxon B. and Turner M., "Fingerprint and iris identification method based on the hough transform," .

[15] Y. Wang Ma L. and T. Tan, "Iris recognition using circular sysmmetric filters," in ICPR.

[16] Petrou M. and P. Bosdogianni, Image Processing: The Fundamentals, John Wiley \& Sons, Chichester, 1999.

[17] Gonzalez R.C. and R.E Woods, Digital Image Processing, Addison-Wesley Publishing Company, 2002.

[18] V. Hlavac Sonka M. and R. Boyle, Image Processing: Analysis and Machine Vison, PWS Publishing Company, 1999.

[19] D. H. Ballard, "Generalizing the hough transform to detect arbitrary shape," Pattern recognition, vol. 13, no. 2, pp. 111122, 1981.

[20] Y. Wang Lim K. and T. Tan, "Iris recognition based on multichanel gabor filtering," in Fifth Asian Conference on Computer Vision, 2002, vol. 1, pp. 279-283.

[21] Mallat S., "A theory of multiresolution signal decomposition," IEEE Trans. on Pattern Analysis and Machine Inteligence, vol. 11, no. 7, pp. 674-693, 1989.

[22] F. Murtagh Starck J.-L. and A. Bijaoui, Image Processinga and Data Analysis: The Multiscale Approach, Cambridge University Press, 1998.

[23] Kong W.K. and D. Zhang, "Detecting eyalash and reflection for accurate iris segmentation," International Journ. of Patt. Rec. and and Artificial Intell., vol. 17, no. 6, pp. 1025-1034, 2003. 